\title{
Changes in knee joint biomechanics following balance and technique training and a season of Australian football
}

\author{
Cyril J Donnelly, ${ }^{1}$ Bruce C Elliott, ${ }^{1}$ Tim L A Doyle, ${ }^{1}$ Caroline F Finch, ${ }^{2}$ \\ Alasdair R Dempsey, ${ }^{1,3}$ David G Lloyd, ${ }^{1,4}$
}

${ }^{1}$ School of Sport Science, Exercise and Health, The University of Western Australia, Perth, Australia ${ }^{2}$ Monash Injury Research Institute, Monash University School of Chiropractic and Sports Science, Murdoch University, Perth, Australia Melbourne, Australia

${ }^{3}$ School of Chiropractic and Sports Science, Murdoch University, Perth, Australia ${ }^{4}$ Centre for Musculoskeletal Research, Griffith University, Gold Coast, Australia

\section{Correspondence to}

Cyril J Donnelly, The University of Western Australia, School of Sport Science, Exercise and Health, 35 stirling Hwy, M408, Perth, Western Australia 6009, Australia; cyril. donnelly@uwa.edu.au

Received 6 December 2011 Accepted 15 March 2012

\begin{abstract}
Purpose Determine if balance and technique training (BTT) implemented adjunct to normal Australian football (AF) training reduces external knee loading during sidestepping. Additionally, the authors determined if an athlete's knee joint kinematics and kinetics change over a season of AF.

Methodology Eight amateur-level AF clubs $(n=1,001$ males) volunteered to participate in either 28 weeks of BTT or a 'sham' training (ST) adjunct to their normal preseason and regular training. A subset of 34 athletes (BTT, $n=20 ; S T, n=14$ ) were recruited for biomechanical testing in weeks $1-7$ and 18-25 of the 28-week training intervention. During biomechanical testing, participants completed a series running, preplanned (PpSS) and unplanned sidestepping (UnSS) tasks. A linear mixed model $(\alpha=0.05)$ was used to determine if knee kinematics and peak moments during PpSS and UnSS were influenced by BTT and/or a season of AF.

Results Both training groups significantly $(p=0.025)$ decreased their peak internal-rotation knee moments during PpSS, and significantly ( $p=0.022)$ increased their peak valgus knee moments during UnSS following their respective training interventions.
\end{abstract}

Conclusions BTT was not effective in changing an athlete's knee joint biomechanics during sidestepping when conducted in 'real-world' training environments. Following normal AF training, the players had different changes to their knee joint biomechanics during both preplanned and unplanned sidestepping. When performing an unplanned sidestepping task in the latter half of a playing season, athletes are at an increased risk of ACL injury. The authors therefore recommend both sidestepping tasks are performed during biomechanical testing when assessing the effectiveness of prophylactic training protocols.

\section{INTRODUCTION}

Anterior cruciate ligament (ACL) injuries in sport are common ${ }^{1}$ and associated with high financial and personal cost. In New Zealand and Australia, ACL injuries cost their respective healthcare systems approximately 17.4 million $\mathrm{NZD}^{1}$ and 75 million $\mathrm{AUD}^{2}$ per year. Following an ACL injury, over $50 \%$ of athletes are not capable of returning to the same level of competition 2 years postreconstruction, ${ }^{3}$ a percentage that increases to approximately $70 \%$ after 3 years. ${ }^{4}$

One-half of non-contact ACL injuries occur during sidestepping sport tasks. ${ }^{5}$ Biomechanical analysis of sidestepping shows that compared with straight-line running, peak extension knee moments are similar, while internal rotation and/ or valgus knee moments are elevated; ${ }^{6-8}$ the same loading patterns that elevate ACL strain measured in cadaveric knee models. ${ }^{9}$ Peak in vivo ACL strain during sporting tasks characterised by a rapid deacceleration phase, ${ }^{10}$ like sidestepping, ${ }^{11}$ generally occur during the weight-acceptance (WA) phase of stance (first $20-30 \%)^{78} 12$ and thought to be when ACL injury risk is the greatest.

The ACL consists of two bundles, the anteromedial bundle (AMB) and posterolateral bundle (PLB), named from their insertions on the tibial plateau. Modelling of the AMB and PLB shows the kinematics of the ACL change as a function of knee flexion angle, with peak elongation observed near full extension. ${ }^{13}$ These results show that knee flexion during stance is associated with ACL injury risk.

Reducing externally applied forces to the ACL can be achieved in two ways. First, reduce the size of the loads applied to the knee by changing an individual's posture or technique during sidestepping. ${ }^{12}{ }^{14-16}$ Second, increase the strength and/or activation of the muscles crossing the knee capable of protecting it when loads are elevated. ${ }^{14} 17$

Training interventions like balance ${ }^{8}$ and technique training ${ }^{15}$ have been shown to be effective in reducing internal rotation and/or valgus knee moments during sidestepping. However, these training interventions have only been shown to be effective when implemented in 'ideal' settings, ${ }^{815}$ which for this study is defined as a training intervention conducted in a controlled laboratory setting, with high athlete compliance $(>80 \%)$ and a low coach to athlete ratio $(<1: 20)$. To date, no study has determined if balance and technique is effective in reducing peak knee loading during sidestepping when implemented in a 'real-world' training environment. This is where training is conducted in a community-level setting with similar coach to athlete ratios as observed during normal training and the instruction is given by a trainer blinded to the intended aims and outcome measures of the intervention.

The primary purpose of this investigation was to determine if balance and technique training (BTT), implemented adjunct to normal preseason and regular season AF training reduces peak knee moments and/or influenced an athlete's knee flexion angle during the WA phase of preplanned 
(PpSS) and unplanned (UnSS) sidestepping. Additionally, we determined if an athlete's knee joint biomechanics change over a season of AF. With this information, we can establish if positive laboratory-based training outcomes can be translated to 'real-world' community-level training environments, and if an athlete's ACL injury risk changes over a playing season.

\section{METHODS}

This study was approved by the Human Research Ethics Committees at The University of Western Australia (UWA) and the University of Ballarat.

\section{Participant population - training intervention}

As part of the Preventing Australian Football Injuries through Exercise (PAFIX) study, ${ }^{19}$ eight Western Australian Amateur Football League (WAAFL) clubs ( $n=1,001$ males) participated in either 28 weeks of BTT or a 'sham' training (ST) intervention adjunct to their 2007 or 2008 preseason and regular season training. The ST intervention served as the experimental control group. All participants provided their informed, written consent before participating in their respective training interventions.

\section{Participant population - biomechanical testing}

From an alphabetical list of the eligible WAAFL participants $(n=1,001) 58$ athletes were recruited via a phone interview by an independent researcher 1 week before training through the first 7 weeks of training (weeks 1-7) for biomechanical testing. Thirty-four (59\%) were available for follow-up testing in weeks 18 to 25 (BTT, n=14; ST, n=20) (figure 1). Exclusion criteria for participants included self-reported joint disorders or had undergone an orthopaedic surgical procedure. All participants provided their informed, written consent before biomechanical testing.

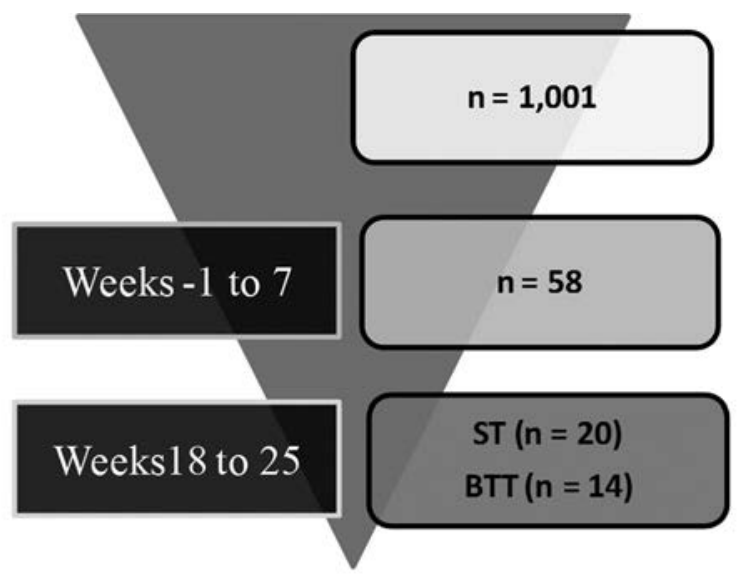

\begin{tabular}{|l|c|c|c|c|}
\hline \multirow{2}{*}{} & \multicolumn{2}{|c|}{ Testing Session 1 (wks-1 to 7) } & \multicolumn{2}{c|}{ Testing Session 2 (wks 18 to 25) } \\
\cline { 2 - 5 } & ST $(\mathbf{n}=\mathbf{2 0})$ & BTT (n= 14) & ST (n= 20) & BTT (n= 14) \\
\hline Age (yrs) & $20.9 \pm 3.1$ & $21.0 \pm 3.3$ & $21.1 \pm 3.1$ & $21.3 \pm 3.2$ \\
\hline Height (m) & $1.84 \pm 0.08$ & $1.86 \pm 0.08$ & $1.83 \pm 0.07$ & $1.86 \pm 0.07$ \\
\hline Mass (kg) & $80.6 \pm 10.2$ & $81.2 \pm 10.0$ & $80.4 \pm 9.8$ & $80.7 \pm 10.6$ \\
\hline
\end{tabular}

Figure 1 Experimental data flow of training intervention and biomechanical testing sessions 1 and 2. BTT and ST numbers were only reported in testing session two as the biomechanists conducting the data collection were blinded to the training intervention codes of each participant until the analysis phase. Mean \pm SD age, body mass and height were reported for participants who completed both testing session 1 and 2.

\section{Training protocol}

Two independent research assistants blinded to (1) which training programs they were overseeing, and (2) the outcome variables analysed during biomechanical testing were assigned to each of the eight WAAFL clubs. Each club consisted of three teams (grade A, B and C), with approximately 25-30 players per team. This made a trainer to athlete ratio of approximately 1:40 for each club. To run each training session, each club uses a staff consisting of a head coach, assistant coaches and athletic trainers. Our research assistants were used in place of the club's normal athletic trainers to conduct 20 min of either ST or BTT at the beginning of each club's regularly scheduled training sessions. Research assistants were considered qualified to run these training sessions after completing a $20 \mathrm{~h}$ coaching seminar associated with their respected training intervention. These research assistants also accurately recorded athlete participation following each training session. ${ }^{20}$

A WAAFL playing season consists of 8 weeks of preseason training and 20 weeks of regular season training, with two regular season bye weeks, where teams trained but did not play a match. Each club trained twice per week during the preseason and regular season, and played one match a week during the regular season.

Training interventions (BTT or ST) were conducted for 20 min before each team's normal training, twice a week in the 8-week preseason, and during the first 10 weeks of the regular season (18 weeks). Training was then condensed to once per week for weeks 19 through 28 (total training sessions, $n=46$ ). Of the participants that completed both biomechanical testing sessions, athletes in the BTT group attended $45 \pm 22 \%$ of the total training sessions and the ST group attended $51 \pm 33 \%$. A one-way ANOVA showed no differences $(p=0.696)$ in the number of training sessions completed by athletes in the BTT and ST groups.

The BTT protocol used for this study is an extension of previous training methods shown to be effective in decreasing peak knee moments during sidestepping. 815 Balance training included single-leg, wobble board, stability disk and Swiss stability ball balance tasks. Each balance exercise became progressively more difficult from weeks 1 to week 18 with the last 10 weeks of training designed as a maintenance phase. During each training session, when appropriate, athletes were verbally instructed to keep their stance foot close to midline, maintain a controlled vertical trunk posture and increase knee flexion during the stance phase of both sidestepping and landing tasks. Interested readers can obtain a detailed description of the BTT training protocol from the corresponding author.

The primary goal of the ST intervention was for athletes to concentrate on improving their acceleration during straightline running tasks, which to our knowledge has not been shown to significantly decrease peak knee joint change to loading or ACL injury rates following training. Neither technique instruction nor balance tasks were included in the ST intervention. The difficulty of the exercises used in the ST intervention progressed with difficulty in a similar fashion to the BTT protocol. Again, interested readers can obtain a detailed description the ST protocol from the corresponding author.

\section{Biomechanical testing}

To evaluate the influence of BTT and a season AF on an athlete's knee joint biomechanics, testing was conducted on two occasions. The first was in weeks 1-7 of the WAAFL preseason training schedule. The second was conducted in weeks 18-25 of their respective training interventions. 

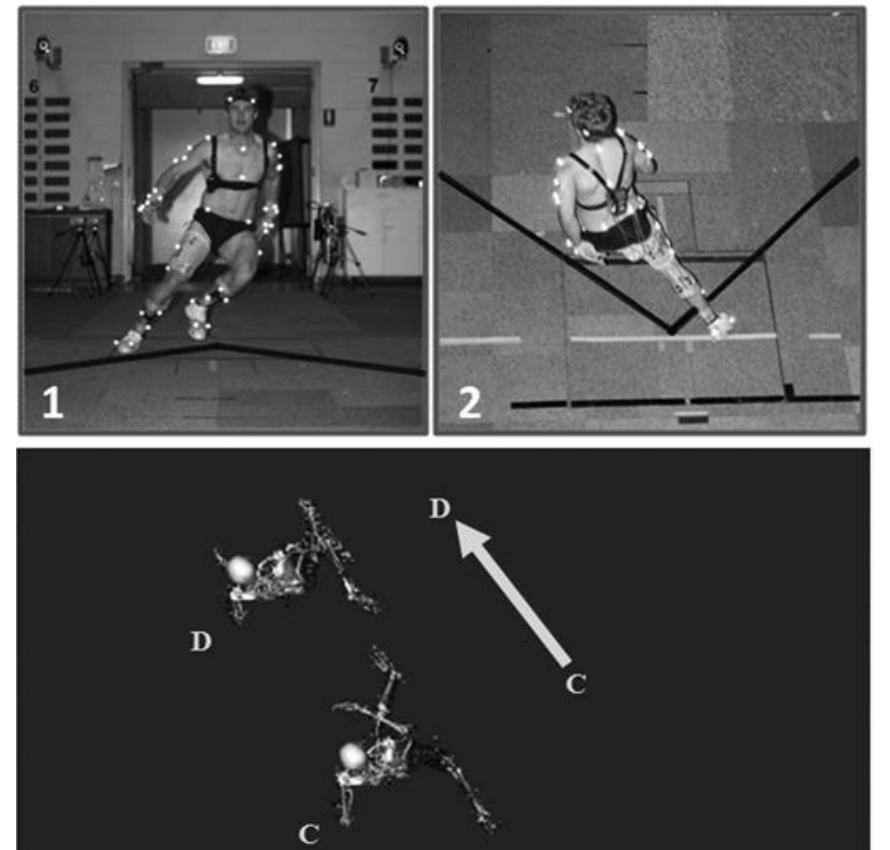

C

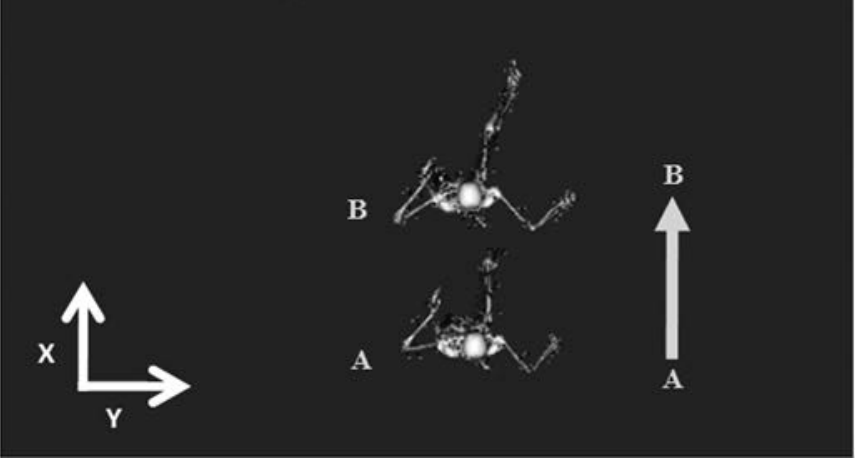

Figure 2 Above: frontal (1) and transverse (2) view of the sidestep sport manoeuvres conducted during biomechanical testing. The solid black lines were used as direction cues for participants during change of direction tasks. Below: mid pelvis position $(x, y)$ coordinates 50 frames prior to heel contact $(A)$, at heel contact $(B)$, contralateral leg heel contact (C) and ipsilateral leg mid swing (D) were used to define vectors $A B$ and $C D$. The cosine of the dot product between vectors $A B$ and $C D$ represents a participants $\mathrm{CoD}$ angle during sidestepping.

During biomechanical testing, participants completed a random series of preplanned and unplanned straight-run, crossover-cut and sidestep running tasks with their self-selected preferred stance limb (figure 2). ${ }^{6-8} 121516$ A computer monitor displayed a $30 \mathrm{~cm}$ arrow to direct participants to perform the straight-run or change of direction running tasks. During unplanned running tasks, the direction arrows were triggered by the athlete running through timing gates situated along the approach pathway. The direction arrow was signalled by the timing gates when participants were approximately $1.5 \mathrm{~m}$ from the force plate, which corresponded to contralateral leg toe off. For all running tasks, a trial was considered successful if the average approach velocity of the right anterior superior iliac spine marker calculated in Vicon workstation (Vicon Peak, Oxford Metrics, UK) was between $4.5 \mathrm{~ms}^{-1}$ and $5.5 \mathrm{~ms}^{-1}$. A successful change of direction trial also required participants to contact a black line marked on the running surface $45^{\circ}$ relative to global $\mathrm{x}$-axis of the laboratory with the contralateral leg during cutting manoeuvre (figure 2). Participants were required to complete three successful trials of each running task before testing was completed. To minimise participant fatigue during the testing period, participants were restricted to maximum of 30 running trials during testing and were given at least $60 \mathrm{~s}$ of rest between each running task.

A 12-camera $250 \mathrm{~Hz}$ Vicon MX motion capture system (Vicon Peak, Oxford Metrics, UK) was used to record three-dimensional full-body kinematics. ${ }^{15} 16$ Ground reaction forces (GRF) were synchronised and recorded at 2000 $\mathrm{Hz}$ from a single $1.2 \times 1.2 \mathrm{~m}$ force plate (Advanced Mechanical Technology, Watertown, Massachusetts, USA).

Ankle joint centres were defined using anatomical landmarks on the medial and lateral malleoli. A six-marker pointer was used to digitise the medial and lateral femoral condyles, with a functional knee axis to define knee joint centres and knee axes orientation. ${ }^{21}$ A functional method was also used to define the hip joint centres. ${ }^{21}$ A custom foot alignment rig was used to measure calcaneous inversion/eversion and foot abduction/adduction to define the anatomical coordinate system of the foot segment. ${ }^{21}$

Marker trajectories and GRF data were both low pass filtered at $15 \mathrm{~Hz}$ using a zero-lag fourth-order Butterworth filter, which was selected based on a residual analysis ${ }^{22}$ and visual inspection.

\section{Analysis}

Spatial-temporal, knee kinematic and knee kinetic variables were analysed during preplanned running (PpRun), PpSS and UnSS sport manoeuvres. Spatial-temporal variables included mean precontact $(\mathrm{PC})$ velocity, mean change of direction (CoD) angle and mean CoD velocity. The PC velocity was calculated as the mean mid-pelvis horizontal velocity $50 \mathrm{~ms}$ prior to heel contact. The CoD angle was calculated by taking the cosine dot product of two vectors representing the position of (a) midpelvis 50 frames before heel contact to (b) heel contact and (c) contralateral leg heel contact to (d) ipsilateral leg mid swing (figure 2). The CoD velocity was determined from the midpelvis resultant velocity during the first $3 / 4$ of stride. The last quarter of stride was not used as this typically occurred outside the calibrated motion capture volume of the laboratory.

Knee kinematics and kinetics were calculated within the WA phase of stance (heel contact to first trough in vertical GRF vector) using custom lower limb kinematic and inverse dynamic models in Bodybuilder (Vicon Peak, Oxford Metrics, UK). ${ }^{6-8} 121516$ Kinematic variables calculated in this phase included mean knee flexion and knee flexion range of motion (RoM). Kinetic variables included mean peak externally applied flexion, valgus and internal-rotation knee moments. All knee moments were normalised to each participant's total body mass and height.

\section{Statistics}

Biomechanical investigators were blinded to each participants training intervention until final statistics were performed. Only athletes who attended both biomechanical testing sessions were included in the analysis. All variables described in the analysis section were assessed using a linear mixed model $(\alpha=0.05)$ in SPSS 17.0.1 (SPSS, IBM Headquarters, Chicago, Illinois, USA). Factors were time (testing session 1 or 2), training intervention (BTT or ST) and running task (PpRun, PpSS or UnSS). The number of training sessions that each athlete participated in-between biomechanical testing sessions was used as a covariate. An adjusted Sidak post hoc analysis $(\alpha=0.05)$ was used to assess significant main effects and interactions. 
A Pearson's Correlation $\left(\mathrm{R}^{2}\right), 95 \% \mathrm{CI}$ and limits of agreement (LoA) for PC velocity, CoD angle and CoD velocity measures were used to assess the reliability of the UWA sidestepping protocol between biomechanical testing sessions 1 and 2. Preempting the results, no statistical differences in $\mathrm{PC}$ velocity, $\mathrm{CoD}$ angle and $\mathrm{CoD}$ velocity were observed between training groups or biomechanical testing sessions, so were grouped together for the aforementioned correlation and LoA analysis.

\section{RESULTS}

No significant differences in knee kinematic variables were observed between training groups or biomechanical testing sessions for all running tasks (table 1). Mean knee flexion and knee flexion RoM were significantly different between running tasks $(\mathrm{p}<0.001)$. Post hoc analyses showed that peak knee flexion and knee flexion RoM were significantly elevated during both sidestepping tasks when compared with PpRun. Only knee flexion RoM was significantly elevated during UnSS when compared with PpSS.

No significant differences in peak knee flexion, valgus or internal-rotation moments were observed between training groups for all running tasks (table 2). Mean peak knee flexion moments were significantly different between running tasks $(p<0.001)$. Post hoc analysis showed that mean peak knee flexion moments during both sidestepping tasks were significantly larger than PpRun.

An interaction between running task and time in peak valgus knee moments was observed $(p=0.037)$. Post hoc analysis showed that peak valgus knee moments were significantly elevated during both sidestepping tasks when compared with PpRun across both testing sessions. Peak valgus knee moments during UnSS were significantly elevated relative to PpSS in testing session 2 , but not during testing session 1 . Peak valgus knee moments during UnSS significantly increased $(p=0.022)$ by $31 \%$ from testing session $1\left(0.48 \pm 0.27 \mathrm{Nm} \mathrm{kg}^{-1} \mathrm{~m}^{-1}\right)$ to testing session $2\left(0.63 \pm 0.40 \mathrm{Nm} \mathrm{kg}^{-1} \mathrm{~m}^{-1}\right)$.

Table 1 Mean knee flexion angle and range of motion (RoM) during the weight acceptance phase of stance for all running tasks. BTT and ST groups across both testing sessions 1 and 2 were pooled together

\begin{tabular}{lll}
\hline & Mean knee flexion (deg) & Knee flexion RoM (deg) \\
\hline PpRun & $26.2 \pm 4.6^{*}$ & $19.6 \pm 5.2^{*}$ \\
PpSS & $29.8 \pm 5.4 \dagger$ & $33.0 \pm 6.2 \dagger$ \\
UnSS & $30.0 \pm 5.0 \dagger$ & $35.3 \pm 6.4 \ddagger$ \\
\hline
\end{tabular}

${ }^{*}, t, \neq$ indicates significant Sidak adjusted post hoc difference between independent variables $(p<0.05)(n=34)$. If two independent variables possess the same symbol they are not significantly different from each other.

Table 2 Mean peak flexion, valgus and internal rotation (Int. Rot.) knee moments of both training groups across testing session 1 and 2 for all running tasks

\begin{tabular}{lllll}
\hline & & $\begin{array}{l}\text { Flexion } \\
\left(\mathbf{N m} \cdot \mathbf{k g}^{-1} \cdot \mathbf{m}^{-1}\right)\end{array}$ & $\begin{array}{l}\text { Valgus } \\
\left(\mathbf{N m} \cdot \mathbf{k g}^{-1} \cdot \mathbf{m}^{-1}\right)\end{array}$ & $\begin{array}{l}\text { Int. Rot } \\
\left(\mathbf{N m} \cdot \mathbf{k g}^{-1} \cdot \mathbf{m}^{-1}\right)\end{array}$ \\
\hline $\mathrm{T} 1$ & PpRun & $1.44 \pm 0.39^{*}$ & $0.15 \pm 0.10^{*}$ & $0.15 \pm 0.09^{*}$ \\
& PpSS & $2.14 \pm 0.55 \dagger$ & $0.37 \pm 0.30 \dagger$ & $0.33 \pm 0.36+\S$ \\
& UnSS & $2.16 \pm 0.42 \dagger$ & $0.48 \pm 0.27 \dagger, \S$ & $0.20 \pm 0.15^{*}$ \\
$\mathrm{~T} 2$ & PpRun & $1.34 \pm 0.25^{*}$ & $0.12 \pm 0.08^{*}$ & $0.13 \pm 0.08^{*}$ \\
& PpSS & $2.15 \pm 0.42 \dagger$ & $0.35 \pm 0.27 \dagger$ & $0.18 \pm 0.09^{*}, \S$ \\
& UnSS & $2.08 \pm 0.44 \dagger$ & $0.63 \pm 0.40 \pm, \S$ & $0.15 \pm 0.06^{*}$ \\
\hline
\end{tabular}

$\S$ indicates significant difference over time $(p<0.05)(n=34)$.

${ }^{*}, t, \neq$ indicates significant Sidak adjusted post hoc difference between independent variables $(p<0.05)(n=34)$. If two independent variables possess the same symbol they are not significantly different from each other.
An interaction in peak internal-rotation knee moments was observed between running task and time $(p=0.026)$. Post hoc analysis showed that in testing session 1, PpSS peak internalrotation knee moments were significantly elevated relative to both UnSS and PpRun. In testing session 2, no differences in peak internal-rotation knee moments were observed between running tasks. Peak internal-rotation knee moments during PpSS significantly decreased $(p=0.025)$ by $45 \%$ from testing sessions $1\left(0.33 \pm 0.36 \mathrm{Nm} \mathrm{kg}^{-1} \mathrm{~m}^{-1}\right)$ to testing session 2 $\left(0.18 \pm 0.09 \mathrm{Nm} \mathrm{kg}^{-1} \mathrm{~m}^{-1}\right)$

The UWA sidestepping protocol reliability test (table 3) showed a moderate to strong correlation in CoD angle during both sidestepping tasks $\left(\mathrm{R}^{2} \geq 0.55\right)$. Between testing sessions 1 and 2 , moderate to strong correlations in $\mathrm{PC}$ velocity and CoD velocity were observed during UnSS $\left(R^{2} \geq 0.46\right)$, while moderate to low correlations were observed during PpSS and PpRun $\left(\mathrm{R}^{2} \leq 0.30\right)$. This is likely attributed to the use of laser timing gates to control for velocity during UnSS. The limits of agreement for all velocity measures were all less than $1.0 \mathrm{~ms}$, which can be considered negligible differences.

An interaction between running task and time in PC velocity was observed $(p=0.022)$ (table 4). Post hoc analysis showed that PC velocity was significantly elevated during PpSS relative to UnSS during testing session 1, but not for testing session 2 . The PC velocity during PpRun was significantly elevated relative to both sidestepping tasks in both testing sessions.

\section{DISCUSSION}

Both balance $^{8}$ and technique ${ }^{15}$ training conducted in controlled laboratory settings have been shown to be effective in decreasing internal rotation ${ }^{8}$ and/or valgus ${ }^{8} 15$ knee moments during both PpSS and UnSS. However, to date, no study has determined if BTT-implemented adjunct to normal 'realworld' preseason and regular season training is effective in reducing peak knee moments during the WA phase of PpSS and UnSS. Additionally, it is unknown if an athlete's knee joint biomechanics change over a playing season. The major finding of this study was BTT implemented in a 'real-world' community-level training environment did not change an athlete's laboratory measurements of knee joint biomechanics during either PpSS or UnSS. However, knee moments during both PpSS and UnSS tasks were found to respond differently over the playing season.

The main finding of this study is that 28 weeks of BTT was not effective in reducing external knee moments when implemented adjunct to normal 'real-world' AF training. These results do not align with previous literature. Neuromuscular ${ }^{23}$ and plyometric ${ }^{24}$ training conducted in 'ideal' training settings were both effective in decreasing valgus knee moments during double-leg drop-landing sport tasks. A condensed plyometricbased training protocol implemented adjunct to regular season basketball training was then shown to be effective in reducing peak extension and valgus knee moments during a similar double-leg drop-landing task (Y. S. Lee, personal communication, 19 January 2011). ${ }^{25}$ Chappell and Limpisvasti ${ }^{26}$ showed that 10-15 min of neuromuscular training adjunct to preseason soccer and regular season basketball training was effective in reducing valgus knee moments during double-leg stop-landing task. These significant results were in part attributed to two factors, (1) high athlete compliance, ${ }^{25} 26$ which was as high as $100 \%,{ }^{26}$ and (2) low coach/trainer to athlete ratios during training, which was approximately $3: 11,{ }^{25}$ and $2: 33 .{ }^{26}$ Within this study, we had low athlete compliance $(45 \%)$ and a relatively 
Table 3 Pearson correlation $\left(\mathrm{R}^{2}\right), 95 \% \mathrm{Cl}$ and limits of agreement (LoA) for change of direction (CoD) angle, precontact $(\mathrm{PC})$ velocity and $\mathrm{CoD}$ velocity between testing session 1 and 2 for all running tasks

\begin{tabular}{lllllll} 
& $\begin{array}{l}\text { CoD Angle } \\
\left(\mathbf{R}^{2} ; \mathbf{9 5 \%} \mathbf{C I}\right)\end{array}$ & $\begin{array}{l}\text { PCVelocity } \\
\left(\mathbf{R}^{\mathbf{2}} ; \mathbf{9 5} \% \mathbf{C I}\right)\end{array}$ & $\begin{array}{l}\text { CoD Velocity } \\
\left(\mathbf{R}^{2} ; \mathbf{9 5} \% \mathbf{C I}\right)\end{array}$ & $\begin{array}{l}\text { CoD Angle } \\
(\text { LoA })\end{array}$ & $\begin{array}{l}\text { PC Velocity } \\
\text { (LoA) }\end{array}$ & $\begin{array}{l}\text { CoD Velocity } \\
\text { (LoA) }\end{array}$ \\
\hline PpRun & - & $0.21 ; 0.13$ to 0.70 & - & - & $\pm 0.9 \mathrm{~ms}^{-1}$ & - \\
PpSS & $0.55 ; 0.54$ to 0.86 & $0.30 ; 0.25$ to 0.75 & $0.29 ; 0.24$ to 0.74 & $\pm 6.1^{\circ}$ & $\pm 0.9 \mathrm{~ms}^{-1}$ & $\pm 0.9 \mathrm{~ms}^{-1}$ \\
UnSS & $0.69 ; 0.68$ to 0.92 & $0.46 ; 0.45$ to 0.83 & $0.50 ; 0.47$ to 0.85 & $\pm 6.8^{\circ}$ & $\pm 1.0 \mathrm{~ms}^{-1}$ & $\pm 0.6 \mathrm{~ms}^{-1}$ \\
\hline
\end{tabular}

Table 4 Mean sidestep CoD angle, CoD velocity and PC velocity for both training groups and across all running tasks. PC velocity was reported for testing sessions 1 and 2

\begin{tabular}{llllll}
\hline & & & Testing session 1 & & Testing session 2 \\
\cline { 5 - 6 } & CoD Angle $\left(^{\circ}\right)$ & $\begin{array}{l}\text { CoD Velocity } \\
(\mathbf{m} / \mathbf{s})\end{array}$ & PC Velocity $(\mathbf{m} / \mathbf{s})$ & & PC Velocity $(\mathbf{m} / \mathbf{s})$ \\
\hline PpRun & $1.0 \pm 0.60^{*}$ & $5.4 \pm 1.68^{*}$ & $5.4 \pm 0.50^{*}$ & $5.3 \pm 0.40^{*}$ \\
PpSS & $16.0 \pm 3.16 \dagger$ & $4.6 \pm 0.49 \dagger$ & $5.1 \pm 0.50 \dagger$ & $5.1 \pm 0.42 \dagger$ \\
UnSS & $16.0 \pm 3.21 \dagger$ & $4.4 \pm 0.55 \dagger$ & $4.9 \pm 0.48 \ddagger$ & $5.0 \pm 0.44 \dagger$ \\
\hline
\end{tabular}

${ }^{*}, \dagger, \ddagger$ indicates significant Sidak adjusted post hoc difference between independent variables $(p<0.05)(n=34)$. If two independent variables posses the same symbol they are not significantly different from each other.

high coach to athlete ratio (1:40). These may have been two major limiting factors preventing the positive biomechanical responses observed in laboratory-based studies ${ }^{8} 15$ from being transferred to a 'real-world' community-level training setting.

It is possible that modifying the BTT protocol may have improved athlete compliance, which may have resulted in different biomechanical outcomes following training. Mykleburst et a ${ }^{27}$ modified their balance training protocol midway between a 2-year training intervention based on athlete and coach feedback. Following these changes, they observed reductions in ACL injury rates in the second season of their training protocol. It is evident that future research should give more attention towards addressing an athlete's perceptions of and compliance to biomechanically based ACL injury-prevention protocols. ${ }^{28}$ Of equal importance, a coach's attitudes and beliefs toward an intended ACL injury-prevention programme must also be addressed to ensure the intended benefits are effectively translated to the athlete. ${ }^{29}$ Considering the psychological needs of both athletes and coaches when implementing injury-prevention protocols at the community level will likely increase athlete compliance ${ }^{30}$ and therefore the probability of positive biomechanical outcomes associated with balance ${ }^{8}$ and technique ${ }^{15}$ training being transferred to 'real-world' community-level training environments. ${ }^{18} 2931$

Within-season training effects were observed in this study. For example, following a season of AF, both the BTT and ST groups displayed a $45 \%$ reduction in internal-rotation knee moments with no change to either their valgus or flexion knee moments during PpSS. This could be due to PpSS being a common sport skill within AF, which is performed repeatedly by athletes during normal training ( $\mathrm{h} \times 2$ days per week) and play ( $2 \mathrm{~h} \times 1$ day per week) in both training groups. It is therefore possible that athletes learnt to adopt techniques during their normal AF training and game play that reduced their internalrotation knee moments and ACL injury risk.

Within-season training effects were also observed during UnSS; external valgus knee moments in both the BTT and ST groups increased by $31 \%$ between testing sessions. These results are supported by previous research, which has shown that following 12 weeks of normal AF football training (control group), valgus knee moments increased by $26 \%$ during the WA phase of sidestepping. ${ }^{8}$ It could be argued that differences in $\mathrm{CoD}$ angle and/or velocity between biomechanical training sessions may have contributed to these observed increases. However, PC velocity, CoD angle or CoD velocity during UnSS were all shown to be similar between testing sessions, suggesting that these variables are not associated with the significant increases in valgus knee moments. Results show an athlete may be at increased risk of ACL injury when performing an unplanned sidestep in the latter half of a playing season. Additionally, these results support previous literature and show that unplanned sport tasks are unique factors associated with ACL injury risk. ${ }^{632}$

The ecological validity of the biomechanical testing protocol used in this investigation may have influenced knee loading measurements during biomechanical testing and in turn the non-significant findings associated with the BTT protocol. For example, all running tasks were conducted under non-fatigued conditions; athletes were tested in isolation rather than in a team environment and differences in the running surface between the laboratory and training environment were apparent. However, we should note that previous training interventions, using the same biomechanical methods have been sensitive enough to measure significant changes in valgus knee loading pre to post training. 815 We therefore believe our 'non change' in knee joint biomechanics following BTT were likely associated with the training intervention and not the biomechanical testing protocol.

\section{CONCLUSIONS}

BTT in 'real-world' training environments, adjunct to normal AF training was not effective in changing an athlete's knee joint kinematics or decreasing external knee moments during the WA phase PpSS or UnSS. Knee joint biomechanics respond to normal AF training differently during both preplanned and unplanned sidestepping tasks. When performing an unplanned sidestepping task in the latter half of a playing season, athletes are at an increased risk of ACL injury. Both preplanned and unplanned sidestepping tasks are therefore recommended during biomechanical testing when assessing the effectiveness of prophylactic training protocols. Athlete compliance to training and coach to athlete ratios should be considered when implementing training interventions in 'realworld' community-level training environments.

Acknowledgements The authors thank Kevin Murray and Laura Firth from the UWA Statistical Consulting Group for statistical advice. Dr Dara Twomey provided support to the PAFIX study in her role as the Victorian-based Project Manager.

Contributors C J Donnelly, B C Elliott, T L A Doyle, C F Finch, A R Dempsey, and D $G$ Lloyd have all made substantial contributions to the following: (1) the conception and design of the study, or acquisition of data, or analysis and interpretation of data, 


\section{What this study adds}

- An athlete's knee joint biomechanics changes over a season of play.

- Preplanned and unplanned sport tasks are recommended when assessing the effectiveness of prophylactic training protocols.

- Athlete compliance is an important factor associated with the 'real-world' implementation of prophylactic training protocols.

(2) drafting the article or revising it critically for important intellectual content, (3) and the final approval of the attached manuscript.

Funding Australian National Health and Medical Research Foundation.

Competing interests None.

Patient Consent Obtained.

Provenance and peer review Not commissioned; externally peer reviewed.

\section{REFERENCES}

1. Gianotti SM, Marshall SW, Hume PA, et al. Incidence of anterior cruciate ligament injury and other knee ligament injuries: a national population-based study. J Sci Med Sport 2009:12:622-7.

2. Janssen KW, Orchard JW, Driscoll TR, et al. High incidence and costs for anterior cruciate ligament reconstructions performed in Australia from 2003-2004 to 20072008: time for an anterior cruciate ligament register by Scandinavian model? Scand J Med Sci Sports 2011 Jan 7. doi: 10.1111/j.1600-0838.2010.01253.x

3. Dunn WR, Spindler KP. Predictors of activity level 2 years after anterior cruciate ligament reconstruction (ACLR): a Multicenter Orthopaedic Outcomes Network (MOON) ACLR cohort study. Am J Sports Med 2010;38:2040-50.

4. Roos H, Ornell M, Gärdsell P, et al. Soccer after anterior cruciate ligament injury-an incompatible combination? A national survey of incidence and risk factors and a 7-year follow-up of 310 players. Acta Orthop Scand 1995;66:107-12.

5. Cochrane JL, Lloyd DG, Buttfield A, et al. Characteristics of anterior cruciate ligament injuries in Australian football. J Sci Med Sport 2007;10:96-104.

6. Besier TF, Lloyd DG, Ackland TR, et al. Anticipatory effects on knee joint loading during running and cutting maneuvers. Med Sci Sports Exerc 2001;33: 1176-81.

7. Besier TF, Lloyd DG, Cochrane JL, et al. External loading of the knee joint during running and cutting maneuvers. Med Sci Sports Exerc 2001;33:1168-75.

8. Cochrane JL, Lloyd DG, Besier TF, et al. Training affects knee kinematics and kinetics in cutting maneuvers in sport. Med Sci Sports Exerc 2010;42:1535-44.

9. Markolf KL, Burchfield DM, Shapiro MM, et al. Combined knee loading states that generate high anterior cruciate ligament forces. J Orthop Res 1995;13:930-5.

10. Cerulli G, Benoit DL, Lamontagne M, et al. In vivo anterior cruciate ligament strain behaviour during a rapid deceleration movement: case report. Knee Surg Sports Traumatol Arthrosc 2003;11:307-11.

11. Jindrich DL, Besier TF, Lloyd DG. A hypothesis for the function of braking forces during running turns. J Biomech 2006;39:1611-20.
12. Dempsey AR, Lloyd DG, Elliott BC, et al. The effect of technique change on knee loads during sidestep cutting. Med Sci Sports Exerc 2007;39:1765-73.

13. Wu JL, Hosseini A, Kozanek M, et al. Kinematics of the anterior cruciate ligament during gait. Am J Sports Med 2010;38:1475-82.

14. Lloyd DG. Rationale for training programs to reduce anterior cruciate ligament injuries in Australian football. J Orthop Sports Phys Ther 2001;31:645-54.

15. Dempsey AR, Lloyd DG, Elliott BC, et al. Changing sidestep cutting technique reduces knee valgus loading. Am J Sports Med 2009;37:2194-200.

16. Donnelly CJ, Lloyd DG, Elliott BC, et al. Optimizing whole-body kinematics to minimize valgus knee loading during sidestepping: Implications for ACL injury risk. J Biomech 2012.

17. Lloyd DG, Buchanan TS. Strategies of muscular support of varus and valgus isometric loads at the human knee. J Biomech 2001;34:1257-67.

18. Finch C. A new framework for research leading to sports injury prevention. J Sci Med Sport 2006;9:3-9.

19. Finch C, Lloyd D, Elliott B. The Preventing Australian Football Injuries with Exercise (PAFIX) Study: a group randomised controlled trial. Inj Prev 2009;15:e1.

20. Twomey DM, Finch CF, Doyle TL, et al. Level of agreement between field-based data collectors in a large scale injury prevention randomised controlled trial. J Sci Med Sport 2011;14:121-5.

21. Besier TF, Sturnieks DL, Alderson JA, et al. Repeatability of gait data using a functional hip joint centre and a mean helical knee axis. J Biomech 2003;36: $1159-68$.

22. Winter DG. Things I've learned about personality from studying political leaders at a distance. J Pers 2005;73:557-84.

23. Myer GD, Ford KR, Palumbo JP, et al. Neuromuscular training improves performance and lower-extremity biomechanics in female athletes. J Strength Cond Res 2005; 19:51-60.

24. Hewett TE, Stroupe AL, Nance TA, et al. Plyometric training in female athletes. Decreased impact forces and increased hamstring torques. Am J Sports Med 1996;24:765-73.

25. Lim BO, Lee YS, Kim JG, et al. Effects of sports injury prevention training on the biomechanical risk factors of anterior cruciate ligament injury in high school female basketball players. Am J Sports Med 2009;37:1728-34.

26. Chappell JD, Limpisvasti 0 . Effect of a neuromuscular training program on the kinetics and kinematics of jumping tasks. Am J Sports Med 2008;36 $1081-6$.

27. Myklebust G, Engebretsen L, Braekken IH, et al. Prevention of anterior cruciate ligament injuries in female team handball players: a prospective intervention study over three seasons. Clin J Sport Med 2003;13:71-8.

28. Finch CF, White P, Twomey D, et al. Implementing an exercise-training programme to prevent lower-limb injuries: considerations for the development of a randomised controlled trial intervention delivery plan. Br J Sports Med 2011:45:791-6.

29. Twomey D, Finch C, Roediger E, et al. Preventing lower limb injuries: is the latest evidence being translated into the football field? J Sci Med Sport 2009;12. 452-6.

30. Deci El, Ryan RM. Intrinsic motivation and self-determination in human behaviour. New York: Plenum Press, pages 11-39, 315-332.

31. Finch CF, Donaldson A. A sports setting matrix for understanding the implementation context for community sport. Br J Sports Med 2010;44:973-8.

32. McLean SG, Borotikar B, Lucey SM. Lower limb muscle pre-motor time measures during a choice reaction task associate with knee abduction loads during dynamic single leg landings. Clin Biomech (Bristol, Avon) 2010;25:563-9. 


\section{BJSM}

\section{Changes in knee joint biomechanics following balance and technique training and a season of Australian football}

Cyril J Donnelly, Bruce C Elliott, Tim L A Doyle, et al.

Br J Sports Med 2012 46: 917-922 originally published online April 30, 2012

doi: 10.1136/bjsports-2011-090829

Updated information and services can be found at:

http://bjsm.bmj.com/content/46/13/917.full.html

These include:

References This article cites 30 articles, 9 of which can be accessed free at: http://bjsm.bmj.com/content/46/13/917.full.html\#ref-list-1

Article cited in:

http://bjsm.bmj.com/content/46/13/917.full.html\#related-urls

Email alerting Receive free email alerts when new articles cite this article. Sign up in service the box at the top right corner of the online article.

Notes

To request permissions go to:

http://group.bmj.com/group/rights-licensing/permissions

To order reprints go to:

http://journals.bmj.com/cgi/reprintform

To subscribe to BMJ go to:

http://group.bmj.com/subscribe/ 